\title{
Estrategias terapéuticas contra el cáncer en animales de compañía
}

\section{Therapeutic strategies against cancer in companion animals}

\author{
Danila de Jesús Ruiz-Castillo, ${ }^{1}$ Erika Arroyo-León ${ }^{2 *}$ y Alejandro Cervantes-Arias ${ }^{3}$
}

Fecha de recepción: 25 de mayo de 2021

Fecha de aceptación: 31 de agosto de 2021

Resumen - El cáncer es una de las principales causas de deceso en perros y gatos. Lo que antes se consideraba una sentencia de muerte ahora es una enfermedad tratable, y en la mayoría de los casos es posible extender tiempos de supervivencia con buena calidad de vida.

El tratamiento antineoplásico suele ser una combinación de múltiples modalidades terapéuticas, en el que la cirugía juega un papel importante para el diagnóstico y control local de la enfermedad. La quimioterapia, y más recientemente la inmunoterapia, contribuye al control sistémico del padecimiento residual. Herramientas como la electroquimioterapia, radioterapia y criocirugía tienen también un lugar en el cuidado de los pacientes oncológicos. La oncología es una rama de la medicina veterinaria aún joven, pero en constante desarrollo en nuestro país.

Palabras clave: Cáncer, quimioterapia, inmunoterapia, cirugía, animal.
Abstract - Cancer is one of the main causes of death in dogs and cats. What was once considered a death sentence is now a disease that can be treated and in most cases, it is possible to extend survival times with good quality of life.

Antineoplastic treatment is usually a combination of multiple therapeutic modalities, in which surgery plays an important role for diagnosis and local control of the disease. Chemotherapy, and most recently immunotherapy, help in the systemic control of the remaining disease. Other modalities such as electrochemotherapy, radiotherapy and cryosurgery can also help in the management of cancer patients.

In Mexico, oncology is still a novel medical specialty, but one in constant development.

Key words: Cancer, chemotherapy, immunotherapy, surgery, animal. 


\section{Introducción}

Dese a que el término cáncer podría hacernos Densar en una enfermedad en específico, la realidad es que engloba a un gran número de padecimientos que pueden iniciar en cualquier órgano o tejido y cuyo desarrollo se basa en el crecimiento celular anormal y no controlado, que puede invadir tejidos vecinos o incluso extenderse a órganos distantes; este último proceso se refiere a la metástasis, que es la principal causa de muerte en pacientes humanos y veterinarios (American Veterinary Medical Association [AVMA], 2021; World Health Organization [WHO], 2021).

El cáncer se puede presentar en animales domésticos, salvajes, aves y peces; las lesiones malignas observadas en ellos son similares a las halladas en humanos, por lo que es importante el estudio de este grupo de enfermedades en poblaciones animales (Dorn, 1967).

En el caso de las pequeñas especies, se estima que uno de cada cuatro perros desarrollará algún tipo de neoplasia en algún momento de su vida, mientras que cerca de la mitad de los perros mayores a 10 años sufrirán algún tipo de cáncer (AVMA, 2021). Se considera que los pacientes caninos desarrollan cáncer a un ritmo similar que en los humanos. En el caso de los gatos, la información es más dispersa (AVMA, 2021).

Actualmente se cuenta con pocas bases de datos que ayuden a generar estadísticas relacionadas con la presencia de cáncer en animales de compañía y México no es la excepción.

El tratamiento antineoplásico en animales de compañía puede ser tan complejo o tan sencillo como cada caso en particular lo requiera. Existen individuos con algún tipo de cáncer que se podría tratar con algo tan simple como una nodulectomía, o bien, hay pacientes que requieren estrategias terapéuticas combinadas, incluyendo cirugía, quimioterapia, radioterapia, inmunoterapia, entre otras.

Asimismo, el cáncer debe ser atacado con base en el estado general de la persona enferma. Sería contraindicado e incluso peligroso establecer que todos los tumores de cierto tipo se deben atender de la misma forma, pues no se está tratando al cáncer, sino a un paciente con cáncer. En la misma línea, podemos enfrentarnos a alguien con cáncer y con alguna 0 algunas enfermedades concomitantes, y es en esos casos en los que con mayor razón debemos entender que estamos tratando a un individuo, no a una enfermedad.

Para poder establecer un protocolo terapéutico individualizado debemos enfatizar en el abordaje diagnóstico de cada paciente. La estadificación desempeña un papel muy importante en la toma de decisiones con respecto al tratamiento a llevar a cabo. Esta estadificación implica determinar la extensión de la enfermedad a un nivel local, regional y distante. Para evaluar dicha extensión podemos apoyarnos desde en la realización de estudios radiográficos de campos pulmonares en sus tres proyecciones y ultrasonido abdominal, hasta en técnicas avanzadas de imagen como una tomografía computarizada (TC) o una resonancia magnética (RMI), a fin de conocer con mayor precisión el tamaño y la extensión de masas grandes, fijas e infiltrativas (evaluación local). La palpalción de linfonodos (LN) es un pobre predictor de metástasis, apoyarse en un estudio citológico evaluado por un patólogo clínico experto será de gran valor; asimismo, detectar los LN en determinadas ubicaciones puede ser difícil, por lo que la linfografía radiográfica o por TC es de mucha utilidad para determinar linfonodos centinela cuando los patrones de drenaje no son claros; también es posible considerar el estudio histopatológico del mismo para evitar subestimar la enfermedad (evaluación regional) (Hauck \& Oblak, 2020).

El diagnóstico es la piedra angular que orientará hacia el tipo de estrategias terapéuticas que podamos incluir en nuestros protocolos. De manera general, estas terapias se dividen en locales y sistémicas: la local es aquella que pretende atacar a un tumor en una localización específica del cuerpo; como ejemplo 
podemos mencionar a la cirugía, la radioterapia, la electroquimioterapia y la criocirugía. Como terapia sistémica definimos a aquellas que intentan inhibir el crecimiento tumoral no solo en un área en particular, sino en todo el organismo; algunos ejemplos son la quimioterapia, la inmunoterapia y la terapia dirigida. A continuación describiremos algunas de ellas de manera individual.

\section{Terapia local}

\section{Cirugía}

La cirugía es una de las principales modalidades terapéuticas en el control del cáncer localizado. La remoción física en estos casos puede curar a más personas que cualquier otra forma de tratamiento $y$ suele ser efectiva en el combate de tumores grandes y localizados (Soderstrom, 1995). Muchos tumores demandan una excisión agresiva por parte del cirujano oncólogo, cuya efectividad depende en su totalidad de la experiencia de éste; "la mejor oportunidad para extirpar un tumor es el primer intento" (Farese et al., 2018).

La cirugía en oncología puede tener diferentes propósitos y es importante establecer las expectativas antes de la misma: prevención, diagnóstico, estadificación, paliación, cura y tratamiento de complicaciones asociadas a las terapias pueden ser los diversos objetivos del procedimiento quirúrgico (Soderstrom, 1995).

En medicina humana, la oncología quirúrgica es una subespecialidad bien definida. De la misma manera, en medicina veterinaria la cirugía se considera la parte más importante del tratamiento en perros y gatos que presentan tumores sólidos; por esta razón, la oncología quirúrgica veterinaria se ha convertido en una especialidad creciente. La Sociedad Veterinaria de Oncología Quirúrgica (The Veterinary Society of Surgical Oncology, VSSO) se fundó en 2006 con la finalidad de promover el arte, la ciencia y la práctica de la cirugía para el tratamiento del cáncer en pacientes veterinarios (Farese et al., 2018).
Existen principios o criterios quirúrgicos descritos por primera vez por el Dr. William Halsted, que consisten en técnica aséptica, separación del tumor del resto del campo quirúrgico, manejo suave del tejido, el cirujano debe evitar el contacto con guantes e instrumentos de zonas ulceradas o abiertas del tumor, disección anatómica aguda del tejido, hemostasia cuidadosa, uso de drenaje en el sitio de resección si se considera necesario, obliteración del espacio muerto y evitar la tensión. Adherirse a estos principios minimiza el riesgo posoperatorio de complicaciones y recurrencia local del tumor, a pesar de que en la actualidad las técnicas quirúrgicas se han vuelto más avanzadas y agresivas (Soderstrom, 1995, Ehrhart \& Culp, 2012; Farese et al., 2018).

\section{Márgenes de escisión}

Se refiere a la dimensión de la resección quirúrgica o "dosis quirúrgica", la cual se clasifica como intralesional (citoreductora), marginal, amplia y radical.

Aplicar mal este criterio es un error frecuente en oncología quirúrgica, ya que por temor a la incapacidad de poder cerrar el defecto resultante, el cirujano aplica una dosis quirúrgica baja, lo que muy probablemente resultará en dejar células tumorales en el sitio (bordes sucios) que más adelante desarrollarán un nuevo tumor de mayor agresividad. Debido a esto, el uso de marcadores quirúrgicos estériles para delinear los márgenes antes de la incisión ayuda al especialista a orientarse y superar la preocupación del cierre de la herida. Estos márgenes quirúrgicos deberán determinarse sobre la base de tipo, comportamiento, ubicación anatómica, grado del tumor y barrera proporcionada por tejido circundante; por ejemplo, se recomienda márgenes laterales de $1 \mathrm{~cm}$ en tumores benignos y de 2 a $3 \mathrm{~cm}$, cuando sea posible, en malignos (Farese et al., 2018).

\section{Resección marginal}

Se realiza solo disecando la periferia de la pseudocápsula tumoral en la zona reactiva. Este 
procedimiento tiene éxito cuando se conoce el tipo de tumor a partir de una biopsia previa; un ejemplo es en tumores benignos como el lipoma. Sin embargo, con los malignos este enfoque no es recomendable (Farese et al., 2018).

\section{Radioterapia}

Otra de las estrategias de tratamiento contra el cáncer se lleva a cabo mediante el uso de radiación ionizante, la cual aniquila directamente a las células tanto malignas como a aquellas que no lo son, debido a sus efectos en el DNA y en menor medida causa daño a las membranas celulares (Moore, 2002).

Además, puede interactuar con el agua intracelular y la matriz intercelular para la formación de radicales libres citotóxicos, acción también conocida como daño indirecto a DNA. Las células afectadas pueden entrar en apoptosis, intentar dividirse y morir; dividirse de forma aberrante; mantenerse funcionales pero incapaces de dividirse; dividirse antes de volverse estériles; presentar alteraciones menores reparables o incluso podrían no presentar alteraciones (Moore, 2002).

Es importante mencionar que de acuerdo con la fase del ciclo en que se encuentre la célula, ésta será más o menos sensible a la radioterapia, siendo las células en mitosis o en fase G2 las más sensibles y las células en G1 o S, las más resistentes (Moore, 2002).

Se debe tener en cuenta que la radioterapia puede inducir daños en los tejidos aledaños a la zona tratada. Los efectos agudos comunes incluyen mucositis, inflamación epidermal húmeda (radiodermitis) y queratitis (Moore, 2002).

La radioterapia está indicada cuando el control local de un tumor sólido no puede obtenerse quirúrgicamente y éste compromete la función, apariencia y calidad de vida del paciente, como en el caso de tumores de cavidad nasal, en la base del corazón y algunos tipos de tumores cerebrales. Asimismo, desempeña un papel en el tratamiento paliativo de cánceres avanzados, endocrinopatías asociadas con adenomas endócrinos y como adyuvante para el linfoma y ciertos tumores benignos (Larue \& Gordon, 2020).

\section{Electroquimioterapia}

La electroquimioterapia es una de las opciones que se han explorado más recientemente para el control del cáncer, sobre todo en el tratamiento de tumores sólidos en los que el tutor de la mascota ha declinado la cirugía o la radioterapia, o cuando esta última no se encuentra disponible.

Es una modalidad que combina la quimioterapia con el principio de la electroporación, empleando campos eléctricos en el tejido durante un tiempo determinado que actúan a través de la membrana plasmática de la célula, dando como resultado cambios en la geometría y las propiedades del tejido. Esta acción provoca que la membrana celular aumente la permeabilidad a agentes que de otra manera no podrían transferirse a su interior (quimioterapéuticos, plásmidos de DNA), generando altas concentraciones intracelulares de los fármacos (Spugnini, 2019; Nemec, 2020).

Además, esta alternativa puede producir efectos antivasculares ("vascular lock") que contribuyen al mantenimiento del quimioterapéutico dentro del tumor (Nemec, 2020). La muerte celular se deriva de la activad citotóxica del quimioterapéutico, de la electroporación irreversible (mínima) (Spugnini, 2019) y de la activación del sistema inmune citotóxico producida por la liberación de antígenos tumorales y patrones moleculares asociados a daño (DAMP's) (Kroemer, 2014).

Los tumores comúnmente tratados con esta estrategia son los sarcomas de tejido blando, carcinomas, tumores perianales y de sacos anales, entre otros (Spugnini, 2019).

\section{Criocirugía}

Esta terapia emplea bajas temperaturas para destruir tejidos malignos o benignos. Existen diferentes técnicas orientadas a desvitalizar el tejido neoplásico al congelarlo, alcanzando temperaturas de $-50^{\circ} \mathrm{C}$ a 
$-60{ }^{\circ} \mathrm{C}$ (Fernandez de Queiroz, 2008). Se indica en lesiones superficiales pequeñas, no invasivas, donde la cirugía se ve limitada por la ubicación anatómica o por la renuencia del paciente a la misma (Murphy, 2001). Se recomienda tratar lesiones menores a 2 cm (Graham, 2001). La tasa de complicaciones es baja, siendo las más frecuentes las transitorias, como edema, dolor y hemorragia. Otras consecuencias de tipo permanente pueden incluir retracción del tejido, neuropatía, ruptura de tendones, entropión y alopecia de la zona tratada (Fernandez de Queiroz, 2008).

\section{Terapia sistémica Quimioterapia}

Uno de los pilares importantes de la terapéutica oncológica es la quimioterapia. Para hablar de ella debemos entender que los protocolos existentes se tienen que utilizar individualmente por paciente. Es posible administrar como terapia única o como terapia combinada con otras estrategias, entre ellas la cirugía. Se clasifica según los medicamentos que se utilicen (monodroga o multidroga) y también con base en el momento en que se aplique, antes o después de otros tipos de tratamiento. La quimioterapia neoadyuvante es aquella que se administra antes de algún otro posible tratamiento y cuyo objetivo generalmente será la citorreducción, para permitir una opción quirúrgica posterior; la quimioterapia adyuvante es la que puede utilizarse después de otro procedimiento y cuyo objetivo general es ampliar el intervalo libre de enfermedad reincidente o metastásica para intentar un tiempo de sobrevida mayor, y la quimioterapia concomitante, que se administra simultáneamente con algún otro procedimiento (por lo regular junto con la radioterapia). La quimioterapia paliativa es la que se elige para pacientes en los que será imposible detener el progreso de la enfermedad y solo se procura mantenerlos con la mejor calidad de vida, por el mayor tiempo posible.

Hay diversas clasificaciones y subclasificaciones de fármacos quimioterapéuticos. A continuación, nos referimos a algunos de ellos.
Agentes alquilantes. Su mecanismo de acción ocurre mediante la unión covalente de grupos alquilo a macromoléculas celulares. En el DNA forman ductos monofuncionales o bifuncionales que generan enlaces cruzados. Ejemplo de estos agentes son ciclofosfamida, melfalán, ifosfamida, clorambucilo, lomustina, dacarbacina, entre otros.

Antibióticos antitumorales. Son productos naturales de fermentación microbiana; producen oxidación y formación de radicales libres de oxígeno que causan la rotura de DNA. Ejemplos son doxorrubicina, mitoxantrona, actinomicina D, antraciclinas y mitomicinas.

Antimetabolitos. Son agentes que inhiben el uso de metabolitos celulares durante el curso de la división y crecimiento celular; generalmente son análogos de compuestos utilizados en el curso normal del metabolismo, específicamente en procesos anabólicos relacionados con la replicación del DNA. Ejemplos de ellos son metotrexato, 5-fluorouracilo, gemcitabina, rabacfosadine (Tanovea) y arabinósido de citocina.

Agentes antimicrotúbulos. Son compuestos alcaloides derivados de la vinca o taxanos. Su mecanismo de acción implica interferencia con la polimerización o despolimerización de los microtúbulos que desempeñan un papel crítico en la división y función celular. Algunos son vincristina, vinblastina, vinorelbina, vindesina, docetaxel y paclitaxel.

Inhibidores de topoisomerasa. Son agentes que inhiben las enzimas topoisomerasa tipo I y || que participan en la separación de la hebra de DNA para su replicación y transcripción. Ejemplo de estos agentes son etopósido y tenipósido (Gustafson \& Bailey, 2020).

\section{Terapia metronómica}

Etimológicamente, la palabra metronómica proviene de metrónomo, que -como sabemos- es un aparato 
para indicar el tiempo o ritmo correcto para que un músico pueda ejecutar adecuadamente una pieza musical. Si extrapolamos este término a la terapia médica, metronómica se refiere precisamente a ese pulso constante de dosis pequeñas, pero a intervalos cortos o ininterrumpidos. Este tipo de terapia se llega a emplear en pacientes cuya enfermedad neoplásica requiere la pulsación de medicamentos para evitar que el cáncer progrese. Diferentes estudios han confirmado que la terapia metronómica puede ralentizar el avance del padecimiento metastásico (Manfred, 2017; Mutsaers \& Biller, 2020). Los mecanismos de acción asociados a la quimioterapia metronómica son cuatro:

- Inhibición de la angiogénesis y vasculogenésis, lo que permite controlar en parte el crecimiento tumoral (Gaspar et al., 2018).

- Regulación de la respuesta inmune, al disminuir el número de células $T$ regulatorias inmunosupresivas (Tregs), las cuales producen citoquinas que suprimen la respuesta citotóxica tumoral específica, así como a las células natural killer (NK) (Gaspar et al., 2017).

- Disrupción de las células madre cancerosas, que tienen el potencial de iniciar el crecimiento de un tumor y de renovación, diferenciación y reparación del DNA. Si bien el mecanismo no está claro, se cree que el efecto antiangiogénico de la quimioterapia metronómica daña el nicho de las células madre cancerosas (Gaspar et al., 2017).

- Dormancia o inactivación tumoral, que es el arresto del ciclo celular. Existen tres tipos de dormancia: angiogénica, inmune y celular, y se piensa que al influir en estas vías la quimioterapia metronómica podría inducir este estado de inactivación tumoral (Gaspar, Henriques, Marconato \& Queiroga, 2017; Argyle, 2020).

\section{Terapia dirigida}

La terapia dirigida se refiere a las sustancias que bloquean el crecimiento y diseminación del cáncer al interferir con moléculas involucradas en esas acciones (Berz, 2011). Un ejemplo puntual de este tipo de terapia es el uso de inhibidores de tirosina quinasa.

\section{Inhibidores de tirosina quinasa}

Las tirosinas quinasas son proteínas que fosforilan otras proteínas en residuos de tirosina; juegan un papel importante en la transducción de señales celulares, regulando el crecimiento celular y la diferenciación (London, 2009). Se ha evidenciado en medicina humana y veterinaria que estas proteínas se activan de manera anormal en tumores malignos (Schemelinin, 2006).

La consecuencia de esta desregulación es la inducción de señalización celular persistente aun en ausencia de regulación negativa apropiada y factores de crecimiento estimulantes, derivando en proliferación celular descontrolada y supervivencia de las células malignas (London, 2009).

Las moléculas que se encargan de bloquear los sitios de unión del ATP de las quinasas se conocen como inhibidores de tirosina quinasa; la unión a estos sitios evita la fosforilación y la subsecuente activación de estos receptores, impidiendo la cascada de señalización celular que llevaría a la proliferación y supervivencia celular (Berz, 2011). Toceranib, masitinib e imatinib son los inhibidores usados más ampliamente en medicina veterinaria. Entre los efectos adversos más comunes se encuentran: anorexia, vómito, diarrea y gastroenterorragia (London, 2009).

\section{Tiglato de tigilanol}

Se aprobó en 2020 su uso en Europa para tratar mastocitomas cutáneos en caninos. El tiglato de tigilanol es un novedosos diterpeno de uso intratumoral que activa a la proteína C quinasa (De Ridder, 2021).

La administración del fármaco produce una respuesta inflamatoria localizada, disrupción de la 
vasculatura tumoral e inducción de la muerte celular por medio de oncosis (Boyle, 2014). Si bien su uso está limitado a mastocitomas en localización con planos anatómicos y de dimensiones específicas, constituye una opción terapéutica más para pacientes con este tipo de neoplasias.

\section{Tanovea ${ }^{\circledR}$-CA1}

\section{(Succinato de Rabacfosadine, VetDC)}

Es un análogo del nucleótido 9 (2-fosfonilmetoxetil) guanina (PMEG), actúa inhibiendo la ADN polimerasa; impide la síntesis de $A D N$, resultando en la detención de la fase $S$ y promoviendo la inducción de apoptosis en células neoplásicas linfoides en perros.

Fue aprobado por la Administración de Alimentos y Medicamentos de los Estados Unidos (FDA) en 2017 como agente único para el tratamiento de linfoma multicéntrico en perros, linfoma cutáneo de linfocitos $T$ en perros, linfoma multicéntrico en perros sin tratamiento previo, mieloma múltiple espontáneo en perros y linfoma recidivante de linfocitos $B$ en perros.

Este producto está solo disponible en Europa y Estados Unidos y es una buena opción para los propietarios que opten por un tratamiento de un único agente, evitando las visitas continuas que requiere el protocolo CHOP con diversos medicamentos convencionales.

Rabacfosadine ha sido usado en combinación alterna con doxorrubicina (Thamm, 2019; Gustafson \& Bailey, 2020) y también en combinación con L-asparaginasa (Cawley et al., 2020).

\section{Inmunoterapia}

El objetivo principal del sistema inmunológico es reconocer y diferenciar lo que es propio de un individuo de aquello que no lo es (Abbas et al., 2018c). La inmunoterapia para combatir el cáncer es un tipo de tratamiento dirigido contra las formas mutadas o transformadas de proteínas celulares que surgen durante la carcinogénesis (conocidas como antígenos tumorales); esta terapia está diseñada para estimular al sistema inmunológico con la finalidad de mejorar o potenciar las respuestas inmunitarias activas contra las células tumorales y así atacarlas mediante la producción de sustancias por parte de células propias o administradas de manera exógena (Barrera, Borbolla \& Arrieta, 2016; Huerta, Luria \& Vega, 2016; Abbas et al., 2018b; Dow \& Guth, 2020).

La inmunoterapia contra el cáncer se puede clasificar en activa y pasiva (Barrera et al., 2016; Gajewski, 2017).

\section{Inmunoterapia activa (específica y no específica)}

Se basa en la liberación de antígenos tumorales a través de diferentes mecanismos para iniciar la captura, procesamiento y presentación de éstos por las células presentadoras de antígenos o APCs. Dentro de esta clasificación encontramos a las vacunas usadas en animales humanos y no humanos (de células dendríticas, de ADN recombinante en vectores, de péptidos y proteínas y la utilización de citocinas) (Barrera et al., 2016).

\section{Inmunoterapia pasiva}

\section{(específica y no específica)}

Consiste en administrar de forma terapéutica anticuerpos monoclonales, con el objetivo de reconocer antígenos que se expresan en la superficie de la célula tumoral. En esta categoría también se engloba el empleo de inhibidores de puntos de control inmunológicos, transferencia adoptiva de linfocitos y transferencia adoptiva de células mononucleares activadas con citocinas (células LAK) (Barrera et al., 2016).

\section{Anticuerpos}

Los anticuerpos (Ac) se producen en las células plasmáticas y están presentes en el suero y en secreciones corporales como el calostro. Son parte esencial de la respuesta inmunológica humoral de los vertebrados, a quienes les brinda protección específica contra los microorganismos con los que un individuo 
estará en contacto natural o artificialmente a lo largo de su vida (Wong Baeza \& Serafín López, 2016; Abbas et al., 2018).

\section{Anticuerpos monoclonales (MAbs)}

Son anticuerpos con una especificidad definida, esto quiere decir que son homogéneos. Pueden reconocer antígenos sobreexpresados en determinados tipos de tumor, bloquean la interacción tumor-estroma o son capaces de promover una respuesta inmunitaria antitumoral (Compte, Ribas \& Sanz, 2015; Wong Baeza \& Serafín López, 2016).

Evolución y sufijos de los anticuerpos monoclonales (Compte Grau et al., 2015):

1. Anticuerpo múrido (omab)

2. Anticuerpo quimérico (ximab)

3. Anticuerpo humanizado (zumab)

4. Anticuerpo humano (umab).

\section{Anticuerpos monoclonales antitumorales}

Se dividen en tres grupos, según la localización de sus dianas:

1. Anticuerpos frente a moléculas expresadas por la célula tumoral: dirigidos principalmente frente a CD19, CD20 y CD30; un ejemplo es rituximab (Compte Grau et al., 2015; Singh et al., 2018).

2. Anticuerpos monoclonales frente a factores de crecimiento: los más comunes son EGF (factor de crecimiento epidermal), HER (receptor de factor de crecimiento epidermal humano), HER2+, PDGFR (receptor de factor de crecimiento derivado de plaquetas) y VEGF (factor de crecimiento vascular endotelial); ejemplo de éstos son cetuximab, trastuzumab, olaratumab y bevacizumab (Compte Grau et al., 2015; Singh et al., 2018).

3. Anticuerpos monoclonales inmunoestimuladores: interactúan con receptores de células efectoras, como los linfocitos T, implicadas en el control de una respuesta inmunitaria, como CTLA-4 y PD-1; ejemplo de ellos son ipilimumab, pembrolizumab y nivolumab (Compte Grau et al., 2015; Singh et al., 2018).

\section{Vacuna contra melanoma}

Actualmente existe una vacuna específica aprobada por el Departamento de Agricultura de Estados Unidos (USDA) para su uso en medicina veterinaria en perros con melanoma, dirigida a la proteína tirosinasa humana, proteína muy similar a la tirosinasa en perros. La vacuna Oncept ${ }^{\circledR}$ incluye ADN plasmídico xenogenético, que contiene el gen que codifica para la tirosinasa humana (huTyr) (Bergman et al., 2006; Regan, Guth, Coy \& Dow, 2016; Klingemann, 2018; Dow \& Guth, 2020).

\section{Interleucina-2}

La interleucina-2 (IL-2) es producida por linfocitos T CD4+, CD8+, células NK y células dendríticas activadas. La IL-2 tiene como principales funciones promover la proliferación, diferenciación y citotoxicidad de linfocitos Th1, Th2, Th17, Treg y células NK. También está involucrada en la eliminación de linfocitos $T$ autorreactivos y apoya la proliferación de linfocitos B.

En 2013, el Comité Europeo de medicamentos de uso veterinario aceptó la suministración del medicamento Oncept ${ }^{\circledR}$ IL-2 (virus de la viruela felina que expresa IL-2 felina recombinante) y en 2015 se permitió en EUA su uso complementario, además de cirugía y radiación en gatos con fibrosarcoma en estadio I (sin metástasis) (Bergman, 2017). 
Tabla 1. Inmunoterapias aprobadas por la FDA en EUA

\begin{tabular}{|c|c|c|c|c|c|c|}
\hline NOMBRE COMERCIAL & NOMBRE DEL COMPUESTO & COMPAÑÍA & INDICACIONES & $\begin{array}{l}\text { ESTATUS REGULATO- } \\
\text { RIO USA (AÑ̃) }\end{array}$ & ESPECIE & $\begin{array}{l}\text { DISPONIBILIDAD } \\
\text { COMERCIAL }\end{array}$ \\
\hline blontress ${ }^{\circledR}$ & $\begin{array}{l}\text { mab linfoma canino } \\
\text { de células b }\end{array}$ & aratana & $\begin{array}{l}\text { linfoma } \\
\text { canino de } \\
\text { células b }\end{array}$ & $\begin{array}{l}\text { USDA licencia } \\
\text { (2015) }\end{array}$ & canino & no \\
\hline na & $\begin{array}{c}\text { vacuna dna linfoma } \\
\text { canino }\end{array}$ & merial/bi & $\begin{array}{l}\text { linfoma } \\
\text { canino de } \\
\text { células b }\end{array}$ & $\begin{array}{l}\text { USDA licencia } \\
\text { condicional } \\
(2015)\end{array}$ & canino & sí \\
\hline na & $\begin{array}{c}\text { vacuna } \\
\text { osteosarcoma } \\
\text { canino con vector } \\
\text { vivo de listeria }\end{array}$ & aratana & osteosarcoma & $\begin{array}{l}\text { USDA licencia } \\
\text { condicional } \\
(2017)\end{array}$ & canino & sí \\
\hline na & $\begin{array}{c}\text { inmunomodulador } \\
\text { de interleucina } 2 \\
\text { felina }\end{array}$ & merial/bi & $\begin{array}{l}\text { fibrosarcoma } \\
\text { en estadio i }\end{array}$ & $\begin{array}{l}\text { USDA licencia } \\
\text { condicional } \\
\text { (2015) }\end{array}$ & felino & sí \\
\hline immunocidin ${ }^{\circledR}$ & $\begin{array}{l}\text { fracción de la } \\
\text { pared celular de } \\
\text { micobacteria }\end{array}$ & novavive & $\begin{array}{l}\text { tumores de } \\
\text { glándula } \\
\text { mamaria }\end{array}$ & $\begin{array}{l}\text { USDA licencia } \\
\text { (2009) }\end{array}$ & canino & sí \\
\hline oncept ${ }^{\circledR}$ & $\begin{array}{l}\text { vacuna dna } \\
\text { melanoma canino }\end{array}$ & merial/bi & melanoma & $\begin{array}{l}\text { USDA licencia } \\
\text { (2010) }\end{array}$ & canino & sí \\
\hline tactress ${ }^{\circledR}$ & $\begin{array}{c}\text { mab linfoma canino } \\
\text { de células t }\end{array}$ & aratana & $\begin{array}{l}\text { linfoma de } \\
\text { células t }\end{array}$ & $\begin{array}{l}\text { USDA licencia } \\
\qquad(2016)\end{array}$ & canino & no \\
\hline tanovea ${ }^{\circledR}$-ca1 & $\begin{array}{l}\text { inyección de } \\
\text { rabacfosadine }\end{array}$ & vetdc & linfoma & $\begin{array}{l}\text { FDA aprobación } \\
\text { condicional } \\
(2017)\end{array}$ & canino & sí \\
\hline
\end{tabular}

Fuente: tabla tomada y modificada de Arroyo, 2021.

Para ejemplificar algunas de las estrategias terapéuticas anteriormente mencionadas, a continuación describiremos brevemente ciertas neoplasias que se presentan con mayor incidencia en animales de compañía en México, mencionando el tipo de tratamiento del cual se podrían beneficiar.

\section{Neoplasias específicas Mastocitoma}

El mastocitoma cutáneo es una de las neoplasias cutáneas más comunes en perros, representando de 7 a 21\% de todas las que se presentan en canes (Stefanello, 2015; Sledge, 2016). No hay predisposición por sexo reportada y la edad media de edad en que surgen es de nueve años.
Las razas más predispuestas son golden retriever y labrador (Misdorp, 2004; Blackwood, 2012; Kiupel, 2017). De acuerdo con el grado, extensión e incluso presencia celular en los bordes quirúrgicos, esta neoplasia se puede atender con diferentes estrategias, empleando cirugía, quimioterapia, electroquimioterapia, terapia dirigida, o bien una combinación de varias de estas opciones terapéuticas.

\section{Linfoma}

El linfoma se encuentra entre las neoplasias diagnosticadas y tratadas más frecuentemente en perros. La más común es la multicéntrica, constituyendo hasta 75\% de los casos; otras menos comunes son la mediastínica, cutánea y hepato-esplénica (Zandvliet, 2016). 
Aunque el linfoma puede afectar a cualquier raza, los perros de talla mediana-grande se encuentran sobrerrepresentados. Tampoco hay predisposición aparente por sexo, pero las hembras no esterilizadas parecen tener menor riesgo (Zandvliet, 2016).

Regularmente, el linfoma se considera como una enfermedad sistémica y no localizada. Es una neoplasia quimiosensible en la mayoría de los casos y si bien la cura es rara, se puede alcanzar un buen control con manejo quimioterapéutico. La cirugía tiene un papel secundario, sobre todo como herramienta diagnóstica (biopsias) y como parte del tratamiento en casos específicos y localizados (ej. linfoma esplénico primario).

\section{Tumor venéreo transmisible (TVT)}

Es un tumor que suele aparecer en genitales, mucosa oral o nasal, y se transmite de forma horizontal, normalmente durante el coito, por la implantación de células tumorales en mucosas (Da Silva, 2014).

Se considera una neoplasia quimiosensible, razón por la cual el tratamiento más efectivo es la quimioterapia sistémica con vincristina, logrando incluso la cura en la mayoría de los casos. Cuando es resistente, se puede considerar la combinación de quimioterapia con cirugía o electroquimioterapia.

\section{Tumores de glándula mamaria}

Los tumores de glándula mamaria son frecuentes en hembras de perros, gatos y humanos (Goldschmidt et al., 2016). La edad promedio en que se presentan es entre 10 y 11 años (Sorenmo, 2003), y las razas con mayor riesgo son poodle y otras pequeñas (Bostock, 1986; Sorenmo, 2003; Goldschmidt, Peña \& Zappulli, 2016).

El manejo terapéutico de este tipo de tumor es en su mayoría quirúrgico, siendo la quimioterapia sistémica adyuvante una opción limitada a pacientes con neoplasias de alto grado o evidencia de metástasis local o a distancia.

\section{Perspectivas a futuro}

Si bien la oncología veterinaria cada vez se desarrolla más en nuestro país, es una rama de la medicina relativamente joven y en constante cambio. Día con día los paradigmas relacionados con el metabolismo, crecimiento tumoral y la resistencia a los quimioterapéuticos van cambiando, y nuevos modelos y teorías emergen.

Herramientas como la inmunoterapia, representada en su mayoría por los anticuerpos monoclonales y las vacunas, al igual que la terapia dirigida, han adquirido un papel muy importante en el tratamiento de los enfermos oncológicos humanos y veterinarios, y sin duda seguiremos hablando de ellas en los próximos años.

A medida que indaguemos y nos documentemos más sobre las particularidades de nuestros pacientes oncológicos, no solo a nivel histopatológico sino también molecular, genético e incluso epigenético, podremos ofrecer más y mejores opciones terapéuticas.

\section{Referencias}

Abbas, A. K., Lichtman, A. H. \& Pillai, S. (2018a). Anticuerpos y antígenos. En Inmunología celular y molecular (pp. 97-116). Elsevier Inc.

Abbas, A. K., Lichtman, A. H. \& Pillai, S. (2018b). Inmunidad antitumoral. En Inmunología celular y molecular (pp. 397-416). Elsevier Inc.

Abbas, A. K., Lichtman, A. H., \& Pillai, S. (2018c). Propiedades y generalidades de las respuestas inmunitarias. En Inmunología celular y molecular (pp. 1-12). Elsevier Ltd.

American Veterinary Medical Association (AVMA). (20 de junio de 2021). Cancer in pets. Recuperado de https://www.avma.org/resources/pet-owners/ petcare/cancer-pets 
Argyle, D. J. (2020). Molecular/Targeted therapy of cancer. En S. Withrow (ed.), Small Animal Clinical Oncology (pp. 251-285). Elsevier Inc.

Arroyo León, Erika. (2021). Inmunoterapia en cáncer de perros y gatos: Anticuerpos monoclonales como terapia complementaria: Estudio de revisión. Tesis de licenciatura no publicada. Universidad Nacional Autónoma de México.

Barrera, L., Borbolla Escoboza, J. R. \& Arrieta Rodríguez, O. G. (2016). Inmunoterapia del cáncer. En Tratamiento del cáncer. Oncología médica, quirúrgica y radioterapia (pp. 185-199). México: Manual Moderno.

Bergman, P. J. (2017). Veterinary Oncology Immunotherapies. Veterinary Clinics of North America-Small Animal Practice, 48(2), 257-277. doi. org/10.1016/j.cvsm.2017.10.004

Bergman, P. J., Camps-Palau, M. A., McKnight, J. A., Leibman, N. F., Craft, D. M., Leung, C., Liao, J., Riviere, I., Sadelain, M., Hohenhaus, A. E., Gregor, P., Houghton, A. N., Perales, M. A. \& Wolchok, J. D. (2006). Development of a xenogeneic DNA vaccine program for canine malignant melanoma at the Animal Medical Center. Vaccine, 24(21), 4582-4585. doi.org/10.1016/j. vaccine.2005.08.027

Berz, D. W. (2011). Targeting the growth factors and angiogenesis pathways: small molecules in solid tumors. Journal of Surgical Oncology, 103(6), 574586. doi: 10.1002/jso.21776.

Blackwood L., M. T. (2012). European consensus document on mast cell tumours in dogs and cats. Veterinary and Comparative Oncology, 10(3), e1-ez9. doi: 10.1111/j.1476-5829.2012.00341.x

Boyle, G. D. (2014). Intra-lesional injection of the novel PKC activator EBC-46 rapidly ablates tumors in mouse models. PLoS One, 9(10), e108887. doi: 10.1371/journal.pone.0108887.

Cawley, J. R., Wright, Z. M., Meleo, K., Post, G. S., Clifford, C. A., Vickery, K. R., Vail, D. M., Bergman, P. J. \& Thamm, D. H. (2020). Concurrent use of rabacfosadine and L-asparaginase for relapsed or refractory multicentric lymphoma in dogs. Journal of Veterinary Internal Medicine, 34(2), 882-889. https://doi.org/10.1111/jvim.15723

Compte Grau, M., Ribas, A. \& Sanz Alcocer, L. (2015). Anticuerpos monoclonales: realidades y perspectivas en el tratamiento del cáncer. En Inmunoterapia del cáncer: Realidades y perspectivas (pp. 21-32). Elsevier Inc.

Da Silva, D. M. (2014). Treatment of canine transmissible venereal tumor using L-asparaginase, prednisone, and surgery in a clinical chemotherapyresistant case. Turkish Journal of Veterinary and Animal Sciences, 38, 220-223. doi:10.3906/vet1309-23.

De Ridder, T. R.-S. (2021). Randomized controlled clinical study evaluating the efficacy and safety of intratumoral treatment of canine mast cell tumors with tigilanol tiglate (EBC-46). Journal of veterinary internal medicine, 35(1), 415-429.

Dorn, C. R. (1967). The epidemiology of cancer in animals. California medicine, 107(6), 481.

Dow, S. \& Guth, A. (2020). Cancer Immunotherapy. En S: Withrow (ed.), Small Animal Clinical Oncology (pp. 231-250). Elsevier Inc.

Ehrhart, N. \& Culp, W. (2012). Principles of surgical oncology. En Veterinary Surgical Oncology (pp. 3-13). Wiley-Blackwell. 
Farese, J. P., Baco, N. J., Liptak, J. M. \& Kow, K. (2018). Introduction to oncologic surgery for the general surgeon. En Veterinary Surgery Small Animal (pp. 325-345). Elsevier Inc.

Fernandez De Queiroz, G. M. (2008). Clinical Study of Cryosurgery Efficacy in the Treatment of Skin and Subcutaneous Tumors in Dogs and Cats. Veterinary Surgery, 37(5), 438-443. doi: 10.1111/j.1532950X.2008.00411.x

Gaspar, T. B., Henriques, J., Marconato, L. \& Queiroga, F. L. (2018). The use of low-dose metronomic chemotherapy in dogs-insight into a modern cancer field. Veterinary and comparative oncology, 16(1), 2-11. doi.org/10.1111/vco.12309

Gajewski, T. F. (2017). Introduction to principles of cancer immunotherapy. En Cancer immunotherapy principles and practice (pp. 231-236). Demos Medical.

Graham, G. F. (2001). Cryosurgery in the management of cutaneous malignancies. Clinical Dermatology, 19(3), 321-327. doi: 10.1016/s0738081x(01)00171-7.

Goldschmidt, M. H., Peña, L. \& Zappulli, V. (2016). Tumors of the mammary gland. Tumors in domestic animals, 723-765. https://doi. org/10.1002/9781119181200.ch17

Gustafson, D. L. \& Bailey, D. B. (2020). Cancer Chemotherapy. En S. Withrow (ed.), Small Animal Clinical Oncology (pp. 182-208). Elsevier Inc.

Hauck, M. L. \& Oblak, M. L. (2020). Tumors of the skin and subcutaneous tissues. En S. Withrow (ed.), Small Animal Clinical Oncology (pp. 352-366). Elsevier Inc.
Huerta Yepez, S., Luria Perez, R. \& Vega, M. I. (2016). Respuesta inmunológica a tumores. En Inmunología molecular, celular y traslacional (pp. 508-525). Wolters Kluwer. Kiupel, M. W. (2011). Proposal of a 2-Tier Histologic Grading System for Canine Cutaneous Mast Cell Tumours to More Accurately Predict Biological Behavior. Veterinary Pathology, 48(1), 147-55. doi: $10.1177 / 0300985810386469$.

Kiupel, M. (2017). Mastcell tumors. En D.J. Meuten, Tumors in domestic animals (pp. 176-193). Wiley Blackwell.

Klingemann, H. (2018). Immunotherapy for dogs: Running behind humans. Frontiers in Immunology, 9, 133. doi: 10.3389/fimmu.2018.00133

Kroemer, G. G. (2014). Immunogenic Cell Death in Cancer Therapy. Annual Review of Immunology, 31, 51-72. doi: 10.1146/annurev-immunol-032712-100008.

Larue, S. M. \& Gordon, I. K. (2020). Radiation Oncology. En S. Withrow (ed.), Small Animal Clinical Oncology (pp. 209230). Elsevier Inc.

London, A. C. (2009). Tyrosine Kinase Inhibitors in Veterinary Medicine. Topics in Companion Animal Medicine, 24 (3), 106-112. doi.org/10.1053/j. tcam.2009.02.002

Manfred, S. (2017). Encyclopedia of Cancer. En Encyclopedia of Cancer (pp. 2809-2812). Springer Reference. doi.org/10.1007/978-3-662-46875-3

Misdorp, W. (2004). Mast cells and canine mast cells tumours. A review. Veterinary Quarterly, 26(4), 156-69. doi: 10.1080/01652176.2004.9695178

Mochel, J. P., Ekker, S. C., Johannes, C. M., Jergens, A. E., Allenspach, K., Bourgois-Mochel, A., Knouse, M., Benzekry, S., Wierson, W., LeBlanc, A. K. \& Kenderian, S. S. (2019). CAR T Cell Immunotherapy in Human and Veterinary 
Oncology: Changing the Odds Against Hematological Malignancies. AAPS Journal, 21(3). doi.org/10.1208/ s12248-019-0322-1

Moore, S. A. (2002). Radiation Therapy for the Treatment of Tumours in Small Companion Animals. The Veterinary Journal, 164(3), 176-187. doi: 10.1053/tvjl.2002.0728

Murphy, S. (2001). Cutaneous Squamous Cell Carcinoma in the Cat: Current understanding and treatment approaches. Journal of Feline Medicine and Surgery, 15(5), 401-407. doi: $10.1177 / 1098612 \times 13483238$

Mutsaers, A. J. \& Biller, B. (2020). Molecular/Targeted Therapy of cancer. En S. Withrow (ed.), Small Animal Clinical Oncology (pp. 251-285). Elsevier Inc.

Nemec, A. M. (2020). Electroporation-Based Treatments in Small Animal Veterinary Oral and Maxillofacial Oncology. Frontiers in Veterinary Science, 7, 575911. doi: 10.3389/ fvets.2020.575911.

Regan, D., Guth, A., Coy, J. \& Dow, S. (2016). Cancer immunotherapy in veterinary medicine: Current options and new developments. The Veterinary Journal, 207,20-28. doi: 10.1016/j.tvjl.2015.10.008.

Schemelinin, I. Š. (2006). Protein Kinases, Their Function and Implication in Cancer and Other Diseases. Folia Biologica, 52(3), 81-100.

Singh, S., Tank, N. K., Dwiwedi, P., Charan, J., Kaur, R., Sidhu, P. \& Chugh, V. K. (2018). Monoclonal antibodies. A review. Current Clinical Pharmacology, 13(2), 85-99. doi.org/10.2174/1574884712666170809124728

Sledge, D. W. (2016). Canine cutaneous mast cell tumours: A combined clinical and pathologic approach to diagnosis, prognosis, and treatment selection. The Veterinary Journal, 215, 43-54. doi: 10.1016/j. tvjl.2016.06.003

Soderstrom, M. J. (1995). Principles of surgical oncology. Veterinary Clinics: Small Animal Practice, 25(1), 97110. doi.org/10.1016/S0195-5616(95)50007-5

Sorenmo, K. (2003). Canine mammary gland tumors. Veterinary Clinics: Small Animal Practice, 33(3), 573596. doi.org/10.1016/S0195-5616(03)00020-2

Spugnini, E.P. (2019). Electrochemotherapy in Veterinary Oncology State-of-the-Art and Perspectives. Veterinary Clinics of North America: Small Animal Practice, 49(5), 967-979. doi: 10.1016/j.cvsm.2019.04.006

Stefanello, D. B. (2015). Comparison of 2- and 3-category histologic grading systems for predicting the presence of metastasis at the time of initial evaluation in dogs with cutaneous mast cell tumors: 386 cases (2009-2014). Journal of the American Veterinary Medical Association, 246(7), 765-769. doi: 10.2460/javma.246.7.765

Thamm, D. H. (2019). Novel Treatments for Lymphoma. Veterinary Clinics of North America - Small Animal Practice, 49(5), 903-915. doi.org/10.1016/j. cvsm.2019.04.004

Wong Baeza, I. \& Serafín López, J. (2016). Anticuerpos. En Inmunología molecular, celular y traslacional (pp. 160-173). Wolters Kluwer.

World Health Organization (WHO). (20 de junio de 2021). Cancer. Recuperado de https://www.who. int/health-topics/cancer\#tab=tab_1

Zandvliet, M. (2016). Canine Iymphoma: a review. Veterinary Quarterly, 36(2), 76-104. doi: 10.1080/01652176.2016.1152633 